\title{
Survey towards Human Activity Recognition using loT Domain
}

\author{
Shreyas Gawande \\ Computer Engineering \\ JSPM's, BSIOTR, Wagholi, Pune, \\ India
}

\author{
Praveda Bansode \\ Computer Engineering \\ JSPM's, BSIOTR, Wagholi, Pune,
}

India

\author{
Jyoti Deshmukh \\ Computer Engineering \\ JSPM's, BSIOTR, Wagholi, \\ Pune, India
}

\author{
Sayali Dukandar \\ Computer Engineering \\ JSPM's, BSIOTR, Wagholi, Pune, \\ India
}

\begin{abstract}
This task presents a novel framework dependent on the Internet of Things (IOT) to Human Activity Recognition (HAR) by observing fundamental signs distantly. Here it is use raspberry pi, wearable sensors, computational psychiatry. Also have used AI calculations to decide the movement done inside four pre-set up classes (walk, climbing and run). With an increased availability in wearable sensors we explore a better understanding of human needs. Then, it can give input during and after the movement is performed, utilizing a distant checking segment with far off perception and programmable alerts. This framework was effectively executed.
\end{abstract}

\section{Keywords}

Raspberry Pi, Location, Activity Detection, Wearable sensors, Machine learning, Deep learning, Long-Short term memory loss, Conditional random field, Computational psychiatry.

\section{INTRODUCTION}

The Internet of Things, IOT, is another idea wherein all detecting items can be associated with the web to have far off and steady admittance to its estimations (information). This entrance takes into account making a move in a quicker manner, with better outcomes and considerably more information included. The information that make these sorts out of frameworks can go from temperature ,open air area, indoor area, stockpiling stock, mugginess or other industry related factors. To put it plainly, any sensor that can be associated with the Internet makes part of an IoT. In such a manner, old style uses of unavoidable processing can be moved up to an IoT conspire for an action acknowledgment application: Human Activity Recognition (HAR). These applications, in their traditional methodology, have been investigated, assessed and created to the point that few generally accessible items have HAR frameworks implicit. This can be seen on some wellness tracker, which have HAR frameworks incorporated into the portable utilizations of their producer. These applications normally enlist and dissect day by day and rest action. The HAR framework comprises of detecting the individual's situating and development, playing out a component extraction and an order of those highlights to choose which action was acted in a pre-chosen rundown of exercises. The HAR frameworks have a few techniques. it presents a few disservices for subjects like exactness, inclusion and cost. Another technique that can beat these difficulties are on-body sensors frameworks or wearable helped HAR . This sort of approach depends on wearable sensors present all through the body, which help to play out the acknowledgment. These gadgets could be those inside another gadget like a PDA.

In this paper, it present a more itemized and novel rendition of a HAR-IOT framework that utilizes a solitary gadget. This framework is planned to be utilized by patients with ongoing heart sicknesses, patients that have their wellbeing status in a non-basic condition yet at the same time need steady checking. The introduced framework centers around the every day schedule, movement and non-intrusive treatment that every quiet should have as a feature of their recovery cycle. Albeit an IOT approach needs a security investigation, our way to deal with the IOT doesn't depend on a remote sensor organization, the information data isn't touchy to outside audience members and, as already stated, the focal point of the paper is to approve the introduced way to deal with HARIoT frameworks. The movement acknowledgment was executed utilizing a classifier that uses the data assembled by this equipment. Two distinct strategies were carried out for the classifiers: Bayes and C4.5. This segment additionally concedes distant admittance to the information and configurable alarms for the action done, if necessary. This paper presents a novel association between a customary HAR framework and an IOT framework with an alternate way to deal with the old style highlight extraction in a HAR framework. This work will be introduced as follows: in Section, the framework engineering is clarified and supplemented with the framework portrayal in Section 3. Area 4 spotlights on clarifying the element extraction technique and the classifier data. The trials and results are introduced.

\section{LITERATURE SURVEY}

\subsection{Wi-Fi based Human activity recognition using Raspberry Pi}

Mix CSI extraction and investigation frameworks utilizing a few wellbeing observing arrangements would address a huge advance forward in this field.

Carried out Human movement acknowledgment framework on the Raspberry and CSI-skilled equipment.[1]

\subsection{Human Suspicious Activity Detection using Deep Learning}

Chosen 5 dubious exercises shaping 5 classifiers and afterward began the cycle of model choice utilizing ResNet. 
ResNet-50 works the best to distinguish dubious exercises occurring.[2]

\subsection{Human Activity recognition using wearable sensors}

Providing accurate and opportune information on people's activities and behaviour is one of the most important tasks in pervasive computing. Innumerable applications can be visualized, for instance, in medical, security, entertainment, and tactical scenarios.[3]

\subsection{Human activity recognition}

Selected 5 suspicious activities forming 5 classifiers and then started the process of model selection using ResNet. ResNet50 works the best to detect suspicious activities happening. [4]

\subsection{Human activity recognition and behavioural prediction using wearable sensors and deep learning}

Psychiatric measurements and new wearable sensors could increase performance in activity detection with sequential and deep classification algorithm. Detect and classify complex activities and differentiate it from other normal life activities using machine and deep learning.[5]

\section{CASE STUDY}

Solution Implemented: For this undertaking it had a go at foreseeing the movement type twoly. In the initial segment I have taken the 561 master designed highlights and executed old style AI models on top of them. In the other part I took the crude gyrator and Body Acceleration perusing and executed Deep learning models on it to attempt to get comparable outcomes to the old style AI models with the master designed highlights.

About Dataset: This dataset is gathered from 30 persons(referred as subjects in this dataset), performing various exercises with a PDA to their abdomens. The information is recorded with the assistance of sensors (accelerometer and Gyroscope) in that advanced cell. This investigation was video recorded to name the information physically.

Overview of dataset: Accelerometer and Gyroscope readings are taken from 30 volunteers(referred as subjects) while performing the following 6 Activities.

\section{Walking 2. Standing 3.Sitting 4. Lying}

Readings are divided into a window of 2:56 seconds with 50\% overlapping. Accelerometer readings are divided into gravity acceleration and body acceleration readings, which has $\mathrm{x}, \mathrm{y}$ and $\mathrm{z}$ components each. Gyroscope readings are the measure of angular velocities which has $\mathrm{x}, \mathrm{y}$ and $\mathrm{z}$ components. Jerk signals are calculated for Body Acceleration readings. Fourier Transforms are made on the above time readings to obtain frequency readings. Now, on all the base signal readings., mean, max, mad, smart, coefficient, energy bands, entropy etc., are calculated for each window. We get a feature vector of 561 features and these features are given in the dataset. Each window of readings is a data point of 561 features.

\section{EXISTING SYSTEM}

The past structures arranged have taken quantifiable methodologies for human movement acknowledgment affirmation. A radio recurrence based way to deal with detecting, Channel State Information (CSI), can utilize minimal expense off-the-rack WiFi equipment. For radio based movement acknowledgment strategies, the remote radio sorts incorporate $\mathrm{ZigBee}, \mathrm{WiFi}, \mathrm{RFID}$, and so on As various radio information may have various characters and handling steps, it generally partition the radio based techniques into four classes: ZigBee radio based movement acknowledgment, WiFi radio based action acknowledgment, RFID radio based action acknowledgment, and other radio based action acknowledgment. In this paper, it is initially present and audit some connected work in every class. At that point, some delegate strategies are contrasted with show their benefits and inconveniences.

Perceiving various human exercises in an Internet-of-Things (IoT) network utilizing differential channel state data (CSI) of the accessible remote constancy (Wi-Fi) signals. Distinctive human exercises in the Wi-Fi climate lead to multipath blurring, bringing about a difference in CSI for every action. This CSI is detected by shrewd IoT gadgets, like advanced mobile phones, tablets, and workstations for movement acknowledgment. The utilization of differential CSI mitigates the balance and foundation. Another benefit of the proposed strategy is that it wipes out the necessity of conventional wearable movement acknowledgment sensors, like gyrator, pedometers, and accelerometers. A long momentary memory (LSTM) model is utilized for programmed highlight extraction and arrangement of human exercises from the differential CSI. Preparing the LSTM model with the period of differential denoised CSI essentially improves the characterization exactness. The outcomes show a decent compromise between model intricacy and order exactness, along these lines guaranteeing better execution when contrasted with the past best in class techniques.

The information is gathered or separated as pulse and pulses from the EEPROM of the wristband to the Raspberry pi board information pins. In the wake of setting the association between Raspberry pi board and wrist band, the board begins getting the sensor information which is changed over into machine Plan of Wearable Prototype Smart Wristband for Remote Health Monitoring 5 language (double structure), at that point changed over these qualities into decimal structure for showing on a chronic screen.

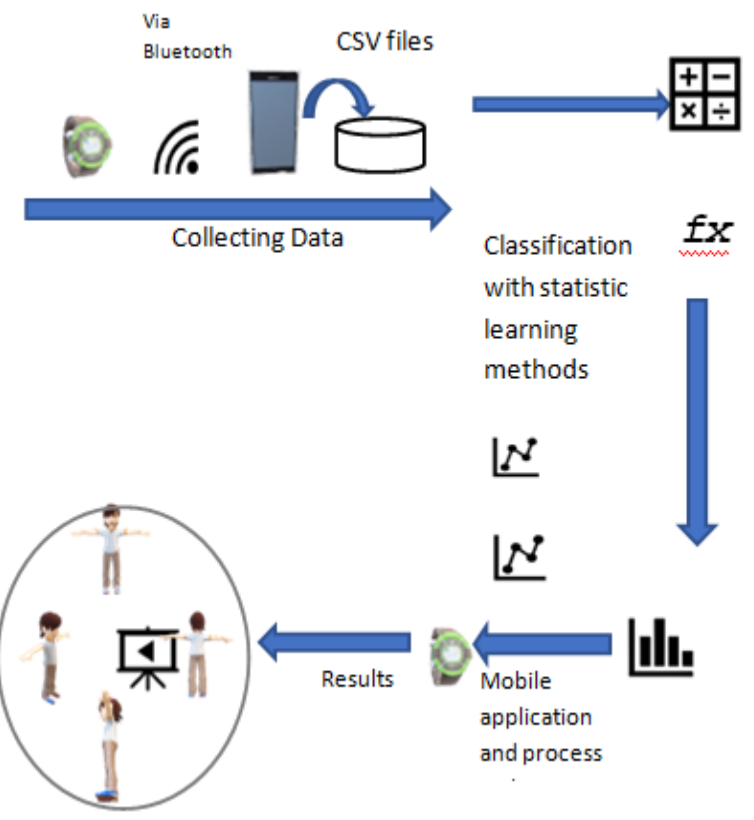

Fig 4.1: Human Activity Recognition 


\section{ROPOSED SYSTEM}

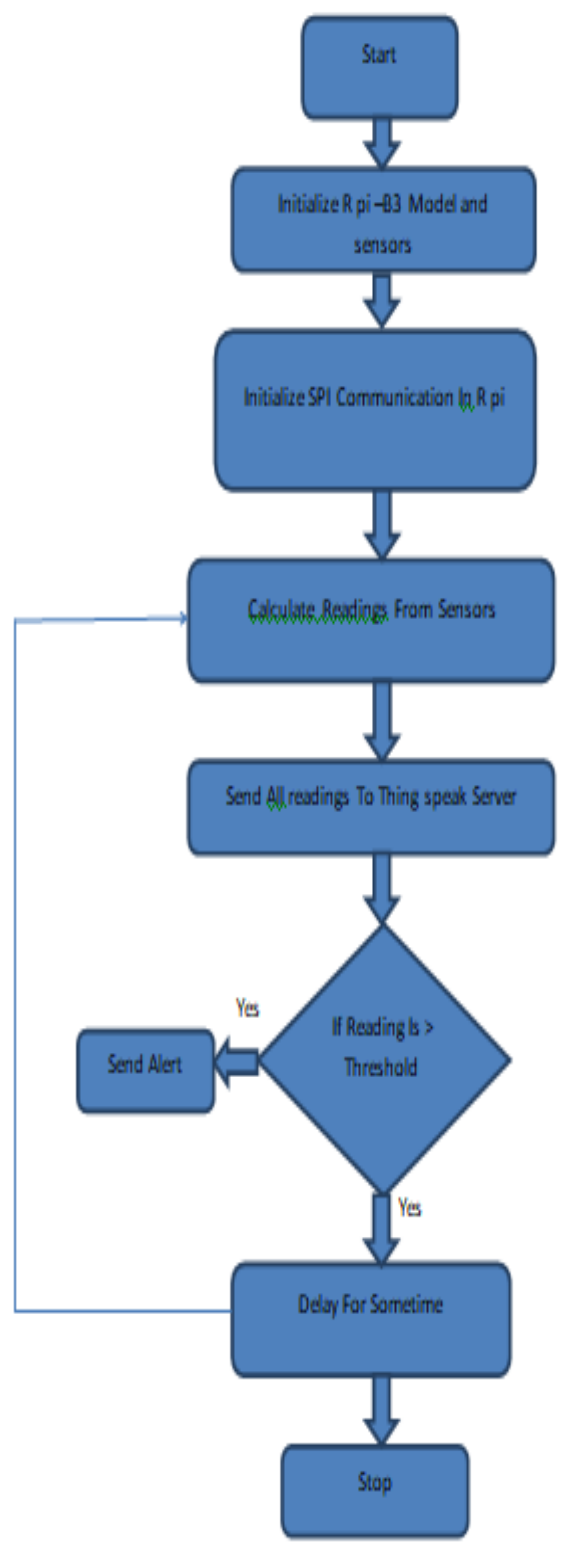

Fig 5.1 Human activity recognition using Raspberry Pi

Description: When the individual began with action the raspberry pi B3 model and those specific sensors will get initiated naturally. They will figure the all readings that are distance, rise, speed, pulse, steps and so on.

This information will be consequently get saved money on the worker. Every one of these estimations are relies upon your web speed and network. Contingent upon that it will give results, it won't give $100 \%$ exactness for any of the estimations. In the event of lost web association all information will be saved money on the worker no information misfortune will be there.

\section{CONCLUSION}

In this study, it proposed a Human Activity Recognition framework utilizing an accelerometer, beat oximeter and Raspberry Pi regulator. It has additionally showed that the past clinical record data can improve the exhibition of the framework. It is thought about 9 exercises, including both basic exercises and day by day exercises and our trial results showed that different exercises can be grouped with an exactness of $95 \%$. Energy productivity can be improved by utilizing the proposed framework as it can precisely anticipate the client's movement, burn-through just the energy needed for the action, and lessening wastage of energy. In addition, the proposed framework can upgrade comfort. For instance, after the framework predicts the client's movement, it can send an admonition to the client if the tally is uncommon.

\section{REFERENCES}

[1] Glean forbes, Stewart Massi, Susan" Wi-Fi based Humanactivity recognition using Raspberry Pi"

[2] G.Vallathan, A.John, SK Mohan "Human Suspicious activity detection using deep learning

[3] G.Vallathan, A.John, SK Mohan"Human Activity recognition using wearable sensors"

[4] Charmi Jobanputra, Jatna Bavishi, Nishant Doshi "Human activity recognition"

[5] Sakron Mekruksavanich "Human activity recognition and behavioural prediction using wearable sensors and deep learning."

[6] M. Billinghurst, "New ways to manage information," The Computer Journal, vol. 32, no. 1, pp. 57-64, 1999.

[7] J. Gubbi, R. Buyya, S. Marusic, and M. Palaniswami, "Internet of Things (IoT): a vision, architectural elements, and future directions," Future Generation Computer Systems, vol. 29, no. 7, pp. 1645-1660, 2013.

[8] L. D. $\mathrm{Xu}, \mathrm{W}$. $\mathrm{He}$, and $\mathrm{S}$. Li, "Internet of things in industries: a survey," IEEE Transactions on Industrial Informatics, vol. 10, no. 4, pp. 2233-2243, 2014.

[9] O. C. Ann and L. B. Theng, "Human activity recognition: A review," in Proceedings of the 4th IEEE International Conference on Control System, Computing and Engineering, ICCSCE 2014, pp. 389-393, mys, November 2014.

[10] Y. LeCun, Y. Bengio, and G. Hinton, "Deep learning," Nature, vol. 521, no. 7553, pp. 436-444, 2015.

[11] Cabra, J.; Castro, D.; Colorado, J.; Mendez, D.; Trujillo, L. An IoT approach for Wireless Sensor Networks Applied to E-Health Environmental Monitoring. In Proceedings of the 10th IEEE International Conference on Internet of Things (iThings 2017), Exeter, Devon, UK, 21-23 June 2017; pp. 14-22.Y

[12] Velasquez, N.; Medina, C.; Castro, D.; Acosta, J.C.; Mendez, D. Design and Development of an IoT System Prototype for Outdoor Tracking. In Proceedings of the International Conference on Future Networks and Distributed Systems-ICFNDS '17, Cambridge, UK, 19-20 July 2017; pp. 1-6.

[13] Fitbit. Heart Rate Tracker: Fitbit Charge 2TM. Available online: https://misfit.com/fitness-trackers/ (accessed on 25 November 2017).

[14] Misfit. Misfit: Fitness Trackers \& Wearable Technology-Misfit.com. Available online: https://www.fitbit. com/home (accessed on 25 November 2017).

[15] Sikder, F.; Sarkar, D. Log-sum distance measures and its application to human-activity monitoring and recognition using data from motion sensors. IEEE Sens. J. 2017, 17, 
$4520-4533$

[16] Testoni, A.; Di Felice, M. A software architecture for generic human activity recognition from smartphone sensor data. In Proceedings of the 2017 IEEE International Workshop on Measurement and Networking (M\&N), Naples, Italy, 27-29 September 2017; pp. 1-6

[17] Boufama, B. Trajectory-Based Human Activity Recognition from Videos. In Proceedings of the 3rd
International Conference on Advanced Technologies for Signal and Image Processing-ATSIP'2017, Fez, Morocco, 22-24 May 2017; pp. 1-5.

[18] Rodriguez, C.; Castro, D.M.; Coral, W.; Cabra, J.L.; Velasquez, N.; Colorado, J.; Mendez, D.; Trujillo, L.C. IoT system for Human Activity Recognition using BioHarness 3 and Smartphone. In Proceedings of the International Conference on Future Networks and Distributed Systems-ICFNDS '17, Cambridge, UK, 19-20 July 2017; pp. 1-7. 